\title{
Interfaces to support collaboration in information retrieval
}

\author{
Michael B. Twidale \& David M. Nichols \\ [mbt|dmn]@comp.lancs.ac.uk \\ Computing Department, Lancaster University \\ http://www.comp.lancs.ac.uk/computing/research/cseg/projects/ariadne/
}

\section{Introduction}

... support for communication and collaboration is as important as support for information-seeking activities and that, indeed, support for the former is needed to support the latter.

[Levy and Marshall, 1995]

Interfaces to information retrieval (IR) systems increasingly support progressive interactive search formulation and refinement. Part of their aim is to make information retrieval easier for the end user without the need for (or with minimal support from) an information intermediary. One approach to supporting these activities has been to design 'intelligent' interfaces that embody some of the knowledge and functions of a human intermediary (e.g. [Belkin and Marchetti, 1990]). An alternative, complementary, approach is to build an interface that actively supports interaction with an intermediary or other user: expanding the 'interactive' nature of the IR system to include other human resources [Hoppe and Zhao, 1994; Twidale et al, 1995].

This social approach, taking direct advantage of human intermediaries' knowledge, in addition to any smart underlying functionality, such as intelligent agents, is clearly more robust and adaptable than solely constructing an 'intelligent' stand-alone interface. It should be generalisable across databases, reactive to system change and likely to become more common as asynchronous interactions dominate accesses to remote digital libraries [Twidale and Nichols, 1996].

However, collaboration has its costs; someone has to pay for the interaction with an expert user, and with large numbers of searchers, help from an expert may be hard to get. Thus it is incumbent on us in advocating collaboration to investigate how it can be supported cost effectively. This will involve minimising the amount of the expert's time that a help session will take (by for example giving the expert a better understanding of the context of the query) and providing mechanisms for the more efficient delivery of the advice constructed. In addition, we wish to investigate how users can minimise their recourse to experts by facilitating the incremental learning of search skills, and by learning from their peers.

In this paper we describe the requirements of interfaces to support these collaborative interactions and our first approach to implementing a system that embodies one kind of explicit support for collaboration, a graphical representation of the search process.

\section{Collaboration in Information Retrieval}

Collaboration already occurs in current physical libraries. However, in addition to the much studied reference interview with a skilled intermediary, substantial informal collaboration occurs. We have observed that many students at Lancaster learn how to use the online public access catalogue (OPAC) from their peers, leaning across adjacent terminals or clustering around a single terminal, engaged in synchronous co-located collaborative learning and working [Twidale, Nichols and Paice, 1996].

This collaboration occurs despite rather than because of the systems, which are conventionally designed on the assumption that users work alone. The collaboration is also desirable, leading to effective learning and reducing the load on information intermediaries. If we are to advocate the development of systems that support collaboration rather than automation, then we must be 
concerned with supporting cost-effective collaboration - allowing users to be able to quickly explain to each other what they have already managed to do and what they now need help on.

The nature of collaborative IR activities will change with the trend towards remote digital libraries and interfaces should support both existing (under-acknowledged) forms of collaboration and the new kinds of collaborative activities opened up by networking.

We are studying the nature of collaboration as it currently occurs in conventional libraries to give us a starting point for developing more advanced systems. To date we have mostly looked at the collaboration that occurs between users, chiefly because this has in the past received far less attention than the expert-novice style interaction of the conventional reference interview. Future work will also look at collaboration between experts such as library staff working together on a user's problem.

\section{Interface Design}

A carefully designed interface offers the potential to provide its users with a visualisation of concepts that are normally only available as abstract ideas. This reification process offers great advantages in facilitating both learning about the concept and cooperative working using the concept.

We undertook a series of preliminary studies [Twidale, Nichols and Paice, 1996] of synchronous remote help giving using a combination of rudimentary tools including Unix talk. It became clear that a crucial need was the ability to share not only the search product (the hits obtained) but also the search process (how those hits were obtained). Observations of the library's help desk backed up this observation; not surprisingly when confronted by users claiming to be unable to find something, librarians frequently ask what they have tried. Equally unsurprisingly, users find this a difficult question to answer: it is very difficult to remember the search actions when one is focusing on the search goals, there is a natural human inclination to auto-correct, remembering what one intended to type rather than what one did, and naturally those most often asking for help are usually the ones who understand the system least and so are most likely to get confused and to lack a specialist vocabulary for describing their actions.

Thus even in a face-to-face help interaction, it is useful to have an interface that enables participants to share an exchangeable representation of the user's search, and indeed perhaps of an expert's proposed solution. Where the user and intermediary are separated by distance and time, such an interface becomes even more important. Although a common representation is needed to aid communication, an expert may benefit from an additional, more powerful view of the information.

One method to achieve this commonality is to use a video-link between users - typically between end-user and library staff [Sugimoto et al, 1995]. However, video is not appropriate for many collaborative activities as it fails to make the search a discrete manipulable object. The C-TORI model of (synchronous or near-synchronous) cooperative IR provides facilities for sharing query histories, cooperatively browsing results and cooperative query formulation [Hoppe and Zhao, 1994]. Nevertheless, the costs and inconvenience of synchronising help or collaboration are likely to override the advantages of its immediacy.

As the search needs to be preserved for communication purposes it can also be used as a visualisation of activity for the end-user. Once a system supports the creation of such an interaction history, it can used in many ways [Hoppe and Zhao, 1994]. Single-user uses include: reuse, error recovery, navigation, reminding and user modelling [Lee, 1992]. Providing a complete record of a search activity frees users from having to remember low-level goalstack details and enables them to concentrate on more strategic elements of the search process. As a digital object it can also be communicated to other people - serving as the basis for a much wider range of collaborative activities [Twidale, Nichols and Paice, 1996]. Such a search object can be stored, high-lighted, edited, annotated, replayed and re-executed: typically the kinds of activities that occur in tutorial and helpgiving interactions [Lemaire and Moore, 1994]. 


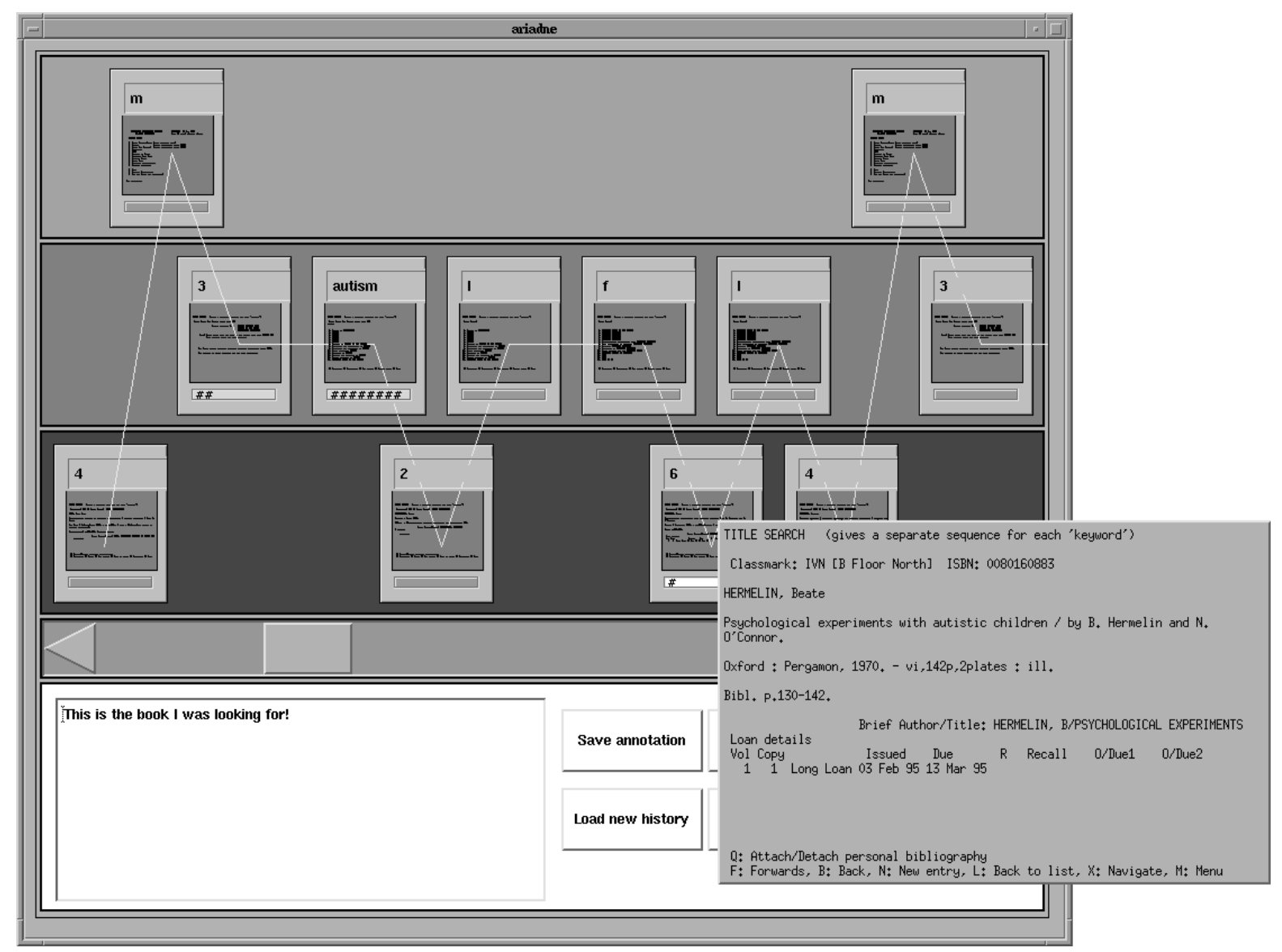

Figure 1 The Ariadne interface

\section{The Ariadne Interface}

We have developed a prototype interface, Ariadne, which attempts to support these activities. Ariadne runs on top of an OPAC or online database, recording the queries and their results and then producing a visualisation of the search process that can be reflected on, shared and discussed by interested parties.

A search is represented as a sequence of cards containing in thumbnail outline the input search command and the output result screen. Cards can be explained by clicking on them. The scrollable sequence of cards can be read left to right as a history of the entire search activity. Additional qualitative information is provided by the vertical position of a card in one of three levels; a high level activity (such as menu selections from the top part of a system's menu hierarchy) is placed on the top level, search queries are placed in the middle level and actions involving the viewing of results on the bottom level. The result is that it is possible to obtain at-a-glance impressions of certain characteristic patterns of behaviour such as the common novice 'error' (dependant upon circumstances) of composing a search that yields hundreds of hits and then proceeding to read through them all. As might be expected, a search session can yield a substantial trace and so facilities are provided to elide sub-sequences of cards, replacing them by a single group card. This card and the individual cards can be annotated, either by the originator of the search or by others commenting upon it.

A main advantage of such an interface is that discussions (both remote and co-located) can then be undertaken between participants about sophisticated searching techniques without the need to learn the specialised vocabulary of the information profession. By contrast, instead of using the abstract 
conceptual terminology, the participants can point to, or highlight, the particular instances that were of significance in the search under discussion.

\section{Conclusion}

We have proposed the development of interfaces for information retrieval systems that are not merely oriented to the delivery of the results of a search, but attempt to support collaboration with other users. These others may be expert intermediaries, co-workers, peer learners, etc. One way of supporting collaboration is to provide a visualisation of the search process that can be manipulated and discussed by the users. We believe that support for cooperative use of an IR system can improve the learning and understanding both of that system and of generic search skills. In addition, by acknowledging the importance of other people in the search process, it leads to a more robust search process, more able to cope with failures of whatever underlying sophisticated intelligent search mechanisms it may additionally use.

\section{Acknowledgements}

Work on the Ariadne project has been funded by the JISC New Technologies Initiative of the HEFCs, and by the British Library Research and Innovation Centre.

\section{References}

Belkin, N.J. \& Marchetti, P.G. (1990), Determining the functionality and features of a intelligent interface to an information retrieval system, Proceedings of the the 13th International Conference on Research and Development in Information Retrieval (SIGIR'90), Brussels, Belgium, ACM.

Hoppe, H.U. \& Zhao, J. (1994), C-TORI: an interface for cooperative database retrieval, in Karagiannis, D. (Ed.), Database and Expert Systems Applications, Berlin: Springer-Verlag, 10313.

Lee, A. (1992), Investigations into History Tools for User Support, PhD Thesis, Department of Computer Science, University of Toronto.

Lemaire, B. \& Moore, J. (1994), An improved interface for tutorial dialogues: browsing a visual dialogue history, Proceedings of the Conference on Human Factors in Computing Systems. CHI'94, Boston, MA, ACM, 16-22.

Levy, D.M. \& Marshall, C.C. (1995), Going digital: a look at assumptions underlying digital libraries, Communications of the ACM 38(4), 77-84.

Sommerville, I., Rodden, T., Sawyer, P., Bentley, R. \& Twidale, M.B. (1993), Integrating ethnography into the requirements engineering process, Proceedings 1st International Conference on Requirements Engineering, San Diego, USA, IEEE Press, 165-74.

Sugimoto, S., Gotou, S., Zhao, Y., Sakaguchi, T. \& Tabata, K. (1995), Enhancing usability of network-based library information system - experimental studies of a user interface for OPAC and of a collaboration tool for library services, Proceedings of Digital Libraries '95, Austin, TX,

Twidale, M.B. \& Nichols, D.M. (1996 to appear), Collaboration and visualisation of the search process, Aslib Proceedings.

Twidale, M.B., Nichols, D.M. \& Paice, C.D. (1996), Browsing is a Collaborative Process, Technical Report CSEG/1/96, Computing Department, Lancaster University.

Twidale, M.B., Nichols, D.M., Smith, G. \& Trevor, J. (1995), Supporting collaborative learning during information searching, Proceedings of Computer Support for Collaborative Learning (CSCL 95), Bloomington, Indiana, 367-74.

Cite as:

Twidale, M.B. and Nichols, D.M. (1996) Interfaces to support collaboration in information retrieval, Proceedings of the BCS Information Retrieval and Human Computer Interaction Workshop, (Eds.) Johnson, C. \& Dunlop, M., Glasgow, UK. 25-28. 